\title{
ON THE ROLE OF FÖLLMER-SCHWEIZER MINIMAL MARTINGALE MEASURE IN RISK-SENSITIVE CONTROL ASSET MANAGEMENT
}

\author{
AMOGH DESHPANDE, * University of Warwick
}

\begin{abstract}
Kuroda and Nagai (2002) stated that the factor process in risk-sensitive control asset management is stable under the Föllmer-Schweizer minimal martingale measure. Fleming and Sheu (2002) and, more recently, Föllmer and Schweizer (2010) observed that the role of the minimal martingale measure in this portfolio optimization is yet to be established. In this paper we aim to address this question by explicitly connecting the optimal wealth allocation to the minimal martingale measure. We achieve this by using a 'trick' of observing this problem in the context of model uncertainty via a two person zero sum stochastic differential game between the investor and an antagonistic market that provides a probability measure. We obtain some startling insights. Firstly, if short selling is not permitted and the factor process evolves under the minimal martingale measure, then the investor's optimal strategy can only be to invest in the riskless asset (i.e. the no-regret strategy). Secondly, if the factor process and the stock price process have independent noise, then, even if the market allows short-selling, the optimal strategy for the investor must be the no-regret strategy while the factor process will evolve under the minimal martingale measure.
\end{abstract}

Keywords: Risk-sensitive control asset management; minimal martingale measure; zero sum stochastic differential game; stability

2010 Mathematics Subject Classification: Primary 49L02

Secondary 60G02

\section{Introduction}

Risk-sensitive control asset management (RSCAM) balances the investor's interest in maximizing the expected growth rate of wealth against his aversion to risk due to deviations of the actually realized rate from the expectation for a finite-time horizon. The subjective notion of investor risk aversion is parameterized by a single variable, say $\theta$. In RSCAM we consider the following criterion to be maximized. For a given $\theta>-2, \theta \neq 0$, and for time horizon $T<\infty$, we find wealth allocation control denoted by $h(t)$, the risk-sensitive expected growth rate up to a time horizon $T$, and criterion $J(v, h, T ; \theta)$ defined by

$$
J(v, h, T ; \theta) \triangleq \frac{-2}{\theta} \log \mathbb{E}\left[\exp \left[\frac{-\theta}{2} \log V^{h}(T)\right]\right],
$$

where $V^{h}(T)$ is the portfolio value at time $T$. An asymptotic expansion around $\theta=0$ for the above criterion yields

$$
J(v, h, T ; \theta)=\mathbb{E}\left[V^{h}(T)\right]-\frac{\theta}{2} \operatorname{var}\left(V^{h}(T)\right)+O\left(\theta^{2}\right), \quad V^{h}(0)=v,
$$

Received 22 March 2013; revision received 26 March 2014.

* Postal address: Department of Statistics, University of Warwick, Coventry, CV4 7AL, UK.

Email address: amogh.deshpande@warwick.ac.uk, addeshpa@gmail.com 
As is obvious from the preceding equation, $\theta>0$ corresponds to a risk-averse investor, $\theta<0$ is a risk-seeking investor, and $\theta=0$ is a risk-neutral investor. Hence, the optimal expected utility function depends on $\theta$ and is a generalization of the traditional stochastic control in the sense that now the degree of risk aversion of the investor is explicitly parameterized through $\theta$ rather than importing it in the problem via an externally defined utility function. For this reason, investment optimization models have been popularly reformulated as risk-sensitive control problems. For a general reference on risk-sensitive control; see Whittle [12].

Risk-sensitive control was first applied to solve financial problems by Lefebvre and Montulet [11] in a corporate finance context and by Fleming [4] in a portfolio selection context. A RSCAM problem with $m$ securities and $n$ (economic) factors was introduced by Bielecki and Pliska [3]. Their factor model, however, made the rather strong assumption that the factor process and the securities price process in their financial optimization model had independent noise. A generalization to this model, relaxing this assumption, was made by Kuroda and Nagai [10], who introduced an elegant solution method based on a change of measure argument which transforms the risk-sensitive control problem into a linear exponential of a quadratic regulator. They solved the associated Hamilton-Jacobi-Bellman (HJB) partial differential equation (PDE) over a finite-time horizon and then studied the properties of the ergodic HJB PDE. We go about formally stating the problem by first describing the factor model for a risk-averse investor.

Let $\left(\Omega, \mathcal{F},\left(\mathcal{F}_{t}\right)_{t \geq 0}, \mathbb{P}\right)$ be the filtered probability space. Consider a market of $m+1 \geq 2$ securities and $n \geq 1$ factors. We assume that the set of securities includes one bond whose price is governed by the ordinary differential equation (ODE)

$$
\mathrm{d} S^{0}(t)=r(t) S^{0}(t) \mathrm{d} t, \quad S^{0}(0)=s^{0},
$$

where $r(t)$ is a deterministic function of $t$. The other security prices are assumed to satisfy the following stochastic differential equations (SDEs):

$$
\mathrm{d} S^{i}(t)=S^{i}(t)\left\{(a+A X(t))^{i} \mathrm{~d} t+\sum_{k=1}^{n+m} \sigma_{k}^{i} \mathrm{~d} W^{k}(t)\right\}, \quad S^{i}(0)=s^{i}, i=1, \ldots, m,
$$

where the component-wise factor process satisfies

$$
\mathrm{d} X^{i}(t)=(b+B X(t))^{i} \mathrm{~d} t+\sum_{k=1}^{n+m} \lambda_{k}^{i} \mathrm{~d} W^{k}(t), \quad X^{i}(0)=x^{i}, i=1, \ldots, n .
$$

Vectorically, $X(t)=\left(X^{1}(t), \ldots, X^{n}(t)\right)^{\top}$ (where the symbol ${ }^{\top}$ signifies transpose) satisfies the following dynamics:

$$
\mathrm{d} X(t)=(b+B X(t)) \mathrm{d} t+\Lambda \mathrm{d} W(t), \quad X(0)=x \in \mathbb{R}^{n} .
$$

Here, $W(t)=\left(W^{k}(t)\right)_{k=1, \ldots, n+m}$ is an $(n+m)$-dimensional standard Brownian motion defined on the filtered probability space. The model parameters $A, B$ are, respectively, $m \times$ $n, n \times n, n \times(m+n)$ constant matrices and $a \in \mathbb{R}^{m}, b \in \mathbb{R}^{n}$. The constant matrix $\left[\sigma_{k}^{i}\right] \triangleq \Sigma, i=1,2, \ldots, m, k=1,2, \ldots,(n+m)$. Matrix $\Sigma \Sigma^{\top}$ is assumed positive definite. Similarly, $\left[\lambda_{k}^{i}\right] \triangleq \Lambda, i=1,2, \ldots, n, k=1,2, \ldots,(n+m)$. Let $|v|$ be a suitable vector norm for any vector $v$ while $\|M\|$ symbolizes a suitable matrix norm for any matrix $M$. As discussed earlier, as part of generalizing the Bielecki and Pliska factor model [3], Kuroda 
and Nagai [10] assumed that the factor process and the securities price process are correlated, i.e. $\Sigma \Lambda^{\top} \neq 0$. The investment strategy which represents proportional allocation of total wealth in the $i$ th security $S^{i}(t)$ is denoted by $h^{i}(t)$ for $i=0,1, \ldots, m$ and we set $S(t):=$ $\left(S^{1}(t), S^{2}(t), \ldots, S^{m}(t)\right)^{\top}, h(t):=\left(h^{1}(t), \ldots, h^{m}(t)\right)^{\top}$, and $g_{t}=\sigma(S(u), X(u) ; u \leq t)$ is the filtration generated by the underlying stock price process and the factor process. Let $\mathscr{H}(T)$ be a space of $\mathbb{R}^{m}$-valued controls for the investor, meaning that we say $h(t) \in \mathcal{H}(T)$, where $h(t)$ are $g_{t}$-progressively measurable stochastic processes such that $\sum_{i=1}^{m} h^{i}(t)+h^{0}(t)=1$, $\mathbb{P}\left(\int_{0}^{T}|h(t)|^{2} \mathrm{~d} t<\infty\right)=1$ and $\mathbb{E}\left[\exp \left(\theta^{2} / 2 \int_{0}^{T} h^{\top}{ }_{t} \Sigma \Sigma^{\top} h_{t} \mathrm{~d} t\right)\right]<\infty$. For given $h(t) \in \mathcal{H}(T)$ the process $V(t)=V^{h}(t)$ is determined by the SDE,

$$
\frac{\mathrm{d} V^{h}(t)}{V^{h}(t)}=h^{0}(t) r(t) \mathrm{d} t+\sum_{i=1}^{m} h^{i}(t)\left\{(a+A X(t))^{i} \mathrm{~d} t+\sum_{k=1}^{m+n} \sigma_{k}^{i} \mathrm{~d} W^{k}(t)\right\}, \quad V^{h}(0)=v,
$$

which can be written vectorically as

$$
\frac{\mathrm{d} V^{h}(t)}{V^{h}(t)}=\left(r(t)+h^{\top}(t) \delta(t)\right) \mathrm{d} t+h^{\top}(t) \Sigma \mathrm{d} W(t), \quad V^{h}(0)=v,
$$

where $\delta(t) \triangleq a+A X(t)-r(t) \mathbf{1}$. From the expression of security and stock price dynamics $S(t)(1.1)$, it is obvious that the market is incomplete (as it has $m$ securities and $n+m$ Brownian drivers) and, hence, there exist many equivalent martingale measures (EMMs). We refer the reader to Karatzas and Shreve [9] for a general treatment on market incompleteness. One such candidate EMM is the Föllmer-Schweizer minimal martingale measure. For the continuousadapted stock price process $S=(S(t))_{0 \leq t \leq T}$, the minimal martingale measure $\mathbb{P}^{*}$ (say) is the unique equivalent local martingale measure with the property that local $\mathbb{P}$-martingale parts of $S$ are also local $\mathbb{P}^{*}$-martingales. For the Föllmer-Schweizer minimal martingale measure $\mathbb{P}^{*}$, the density process is given by the following dynamics:

$$
\frac{\mathrm{d} \mathbb{P}^{*}}{\mathrm{~d} \mathbb{P}}=\mathcal{E}\left(-\int_{0}\left(\left(\Sigma^{\top}\left(\Sigma \Sigma^{\top}\right)^{-1}\right) \delta\right)^{\top} \mathrm{d} W\right)_{T} .
$$

In [10], it was observed that the condition of stability of the matrix $B-\Lambda \Sigma^{\top}\left(\Sigma \Sigma^{\top}\right)^{-1} A$ induces stability on the factor process $X=(X(t))_{0 \leq t \leq T}$ under the minimal martingale measure. Fleming and Sheu [5] and, more recently, Föllmer and Schweizer [6] noted that this observation and, more significantly, the role of the minimal martingale measure in this portfolio asset management problem are yet to be established. In this paper we address these questions. We do so by conceptualizing the RSCAM as a zero sum stochastic differential game between (a market) that provides a probability measure that works antagonistically against another player (the investor) who otherwise wants to maximize the risk-sensitive criterion. We call this game GI; see (2.2). We need to determine the controls that form the saddle point equilibrium to this game. This will then illuminate the explicit dependence between controls $h(t)$ and the probability measure which would then lead us to connect the role played by the minimal martingale measure. We achieve this objective through the following road map.

\subsection{Key steps}

Step 1. We reformulate the game GI into an auxiliary game characterized by the exponential of integral criterion that involves just the factor process $X$. We call this game GII; see (2.7).

Step 2. We then provide a verification lemma for GII. 
Step 3. We then obtain the optimal controls and deduce the connection between the minimal martingale measure and the investor's optimal strategy.

Step 4. To complete the analysis we end by showing that the controls obtained while solving game GII in step 3 in fact also constitute a saddle point equilibrium strategy for the original game GI.

\section{Worst-case risk-sensitive zero sum stochastic differential game}

As discussed in the introduction, the Kuroda and Nagai investment market model is incomplete. We are interested in understanding the influence the minimal martingale measure has on this portfolio optimization problem. We conjure an approach whereby we can explicitly characterize the dependence between the minimal martingale measure and the control variable $h$. Formally, in order to do this, we define a 'market world'. The market world is a space of probability measures defined as

$$
\mathcal{P} \triangleq\left\{\mathbb{P}^{\eta, \xi}:(\eta, \xi)=(\eta(t), \xi(t))_{T \geq t \geq 0} \in \mathcal{O}(T)\right\}
$$

on $(\Omega, \mathcal{F})$, where $\mathcal{O}(T)$ denotes the set of deterministic controls $\eta(t) \in \mathbb{R}^{n \times(n+m)}$ and $\xi(t) \in \mathbb{R}^{1 \times(n+m)}$ which are continuous over the compact set $[0, T]$ and, hence, bounded. For $(\eta(t), \xi(t)) \in \mathcal{O}(T)$ for fixed-time horizon $T$, the restriction of $\mathbb{P}^{\eta, \xi}$ to the $\sigma$-field $\mathcal{F}_{T}$ is given by the Radon-Nikodym density

$$
\left.D^{\eta, \xi}(T) \triangleq \frac{\mathrm{d} \mathbb{P}^{\eta, \xi}}{\mathrm{d} \mathbb{P}}\right|_{\mathcal{F}_{T}} \triangleq \mathcal{E}\left(\int_{0}\left(\eta(t)^{\top} X(t)+\xi^{\top}(t)\right)^{\top} \mathrm{d} W(t)\right)_{T}
$$

with respect to the reference measure $\mathbb{P}$. Here, $\mathcal{E}(\cdot)$ is the Doleáns-Dade exponential. We now show that for $(\eta, \xi) \in \mathcal{O}(T), \mathbb{P}^{\eta, \xi}$ is a probability measure.

Lemma 2.1. Let $\mathbb{E}\left[D^{\eta, \xi}(T)\right]=1$ for all $(\eta, \xi) \in \mathcal{O}(T)$.

Proof. The process $X(t)$ in (1.2) is a Gaussian process. From (1.2) and Grönwall's inequality, we have $\mathbb{E}|X(t)| \leq(\mathbb{E}|X(0)|+|b| T) \exp (\|B\| t)$ and $\operatorname{cov}(X(t))=\Lambda^{\top} \Lambda t$, where $\operatorname{cov}$ is the covariance function. As $\eta(t), \xi(t)$ are deterministic controls and are bounded, $\phi(t) \triangleq X^{\top}(t) \eta(t)+\xi(t)$ is also a Gaussian process with bounded mean and covariance on a finite-time interval $[0, T]$. Hence, by an application of Novikov's condition, the DoleánsDade exponential in (2.1) is a $\mathbb{P}$-martingale. A standard proof of this fact can be found in Bensoussan [1, Lemma 3.1.1].

We now evaluate the optimization criterion $J$ under the new probability measure $\mathbb{P}^{\eta, \xi}$ and call it $\tilde{J}$, which is defined as

$$
\tilde{J}(v, h, \eta, \xi, T ; \theta)=\frac{-2}{\theta} \log \mathbb{E}^{\eta, \xi}\left[\exp \left[\frac{-\theta}{2} \log V^{h, \eta, \xi}(T)\right],\right.
$$

where the portfolio value under the new probability measure $\mathbb{P}^{\eta, \xi}$ is given by

$$
\begin{aligned}
\frac{\mathrm{d} V^{h, \eta, \xi}(t)}{V^{h, \eta, \xi}(t)} & =\left[r(t)+h^{\top}(t)\left(\delta(t)-\Sigma\left(\eta^{\top}(t) X(t)+\xi^{\top}(t)\right)\right)\right] \mathrm{d} t+h^{\top}(t) \Sigma \mathrm{d} W^{\eta, \xi}(t), \\
V^{h, \eta, \xi}(0) & =v .
\end{aligned}
$$


From Lemma 2.1, we have $\mathbb{P}^{\eta, \xi}$ is a probability measure for $(\eta, \xi) \in \mathcal{O}(T)$. From the standard result in Girsanov [8], under the probability measure $\mathbb{P}^{\eta, \xi}$,

$$
W^{\eta, \xi}(t) \triangleq W(t)+\int_{0}^{t}\left(\eta^{\top}(s) X(s)+\xi^{\top}(s)\right) \mathrm{d} s
$$

is a standard Brownian motion process and, therefore, the factor process $X(t)$, vectorically, satisfies the following SDE:

$$
\mathrm{d} X(t)=\left(b+B X(t)-\Lambda\left(\eta^{\top}(t) X(t)+\xi^{\top}(t)\right)\right) \mathrm{d} t+\Lambda \mathrm{d} W^{\eta, \xi}(t) .
$$

Remark 2.1. From (1.4) and (2.1), it is clear that $\mathbb{P}^{\eta, \xi}$ is a minimal martingale measure for $\hat{\eta}(t) \triangleq \eta(t)=A^{\top}\left(\Sigma \Sigma^{\top}\right)^{-1} \Sigma$ and $\hat{\xi}(t) \triangleq \xi(t)=(a-r(t) 1)^{\top}\left(\Sigma \Sigma^{\top}\right)^{-1} \Sigma$.

Kuroda and Nagai [10] stated that under the condition of stability of the matrix $B-\Lambda \Sigma^{\top}\left(\Sigma \Sigma^{\top}\right)^{-1} A$, the factor process $X(t)$ is stable under the minimal martingale measure. In light of our Remark 2.1, we validate this statement now.

Remark 2.2. As $\eta(t)=\hat{\eta}(t)$ and $\xi(t)=\hat{\xi}(t)$ corresponds to the minimal martingale measure, the dynamics of $X(t)$ under the minimal martingale measure can be written as

$$
\mathrm{d} X(t)=\left(b-\Lambda \Sigma^{\top}\left(\Sigma \Sigma^{\top}\right)^{-1}(a-r(t) 1)+\left(B-\Lambda \Sigma^{\top}\left(\Sigma \Sigma^{\top}\right)^{-1} A\right) X(t)\right) \mathrm{d} t+\Lambda \mathrm{d} W^{\hat{\eta}, \hat{\xi}}(t) .
$$

We are interested in finding the behavior of the solution $X(t)$ as $t \rightarrow \infty$. The coefficient of the $X(t)$ term in the drift part of the above equation is $B-\Lambda \Sigma^{\top}\left(\Sigma \Sigma^{\top}\right)^{-1} A$. Since, by assumption, this coefficient term is a stable matrix, $X(t)$ is, hence, stable under the minimal martingale measure.

We need to now pin down the influence the minimal martingale measure has on this portfolio optimization problem to further resolve the inquiry posed by Fleming and Sheu [5]. To do so, as stated earlier, we conceptualize this problem as a game between a player termed as 'the market against the investor'. We denote this game as GI.

Game GI. Obtain $\hat{h} \in \mathcal{H}(T)$ and $(\hat{\eta}, \hat{\xi}) \in \mathcal{O}(T)$ such that

$$
\begin{aligned}
\tilde{J}(v, \hat{h}, \hat{\eta}, \hat{\xi}, T ; \theta) & =\sup _{h \in \mathscr{H}(T)} \inf _{(\eta, \xi) \in \mathcal{O}(T)} \frac{-2}{\theta} \log \mathbb{E}^{\eta, \xi}\left[\exp \left[\frac{-\theta}{2} \log V^{h, \eta, \xi}(T)\right]\right] \\
& =\inf _{(\eta, \xi) \in \mathcal{O}(T)} \sup _{h \in \mathscr{H}(T)} \frac{-2}{\theta} \log \mathbb{E}^{\eta, \xi}\left[\exp \left[\frac{-\theta}{2} \log V^{h, \eta, \xi}(T)\right]\right] .
\end{aligned}
$$

Our intention is to write the objective function $\tilde{J}$ purely in terms of the factor process $X$. We set out to achieve this by defining

$$
g(x, h, \eta, \xi, r ; \theta) \triangleq \frac{1}{2}\left(\frac{\theta}{2}+1\right) h^{\top} \Sigma \Sigma^{\top} h-r-h^{\top}\left(\delta-\Sigma\left(\eta^{\top} x+\xi^{\top}\right)\right) .
$$

Hence, from (2.3), we have

$$
\begin{aligned}
-\frac{\theta}{2} \mathrm{~d} \log V^{h, \eta, \xi}(t)= & \left(\frac{\theta}{2}(X(t), h(t), \eta(t), \xi(t), r(t) ; \theta)-\frac{\theta^{2}}{8} h^{\top}(t) \Sigma \Sigma^{\top} h(t)\right) \mathrm{d} t \\
& -\frac{\theta}{2} h^{\top}(t) \Sigma \mathrm{d} W^{\eta, \xi}(t) .
\end{aligned}
$$


We next define the following stochastic exponential:

$$
\left.\frac{\mathrm{d} \mathbb{P}^{h, \eta, \xi}}{\mathrm{d} \mathbb{P}^{\eta, \xi}}\right|_{\mathcal{F}_{T}}=\mathcal{E}\left(-\frac{\theta}{2} \int_{0} h^{\top}(t) \Sigma \mathrm{d} W^{\eta, \xi}(t)\right)_{T} .
$$

From the definition of the class of controls $\mathscr{H}(T)$, it is clear from an application of Novikov's condition that $\mathbb{P}^{h, \eta, \xi}$ is a probability measure. Under this probability measure $\mathbb{P}^{h, \eta, \xi}$, the standard result of Girsanov [8] yields

$$
W^{h, \eta, \xi}(t) \triangleq W^{\eta, \xi}(t)+\int_{0}^{t} \frac{\theta}{2} \Sigma^{\top} h(s) \mathrm{d} s
$$

is a standard $\mathbb{P}^{h, \eta, \xi}$-Brownian motion and the factor process $X(t)$ satisfies the following dynamics:

$$
\mathrm{d} X(t)=\left(b+B X(t)-\Lambda\left(\eta^{\top}(t) X(t)+\xi^{\top}(t)\right)-\frac{\theta}{2} \Lambda \Sigma^{\top} h(t)\right) \mathrm{d} t+\Lambda \mathrm{d} W^{h, \eta, \xi}(t) .
$$

Now, under the new probability measure $\mathbb{P}^{h, \eta, \xi}$ and using (2.2)-(2.4) and (2.5), we define an auxiliary optimization criterion $I(v, x, h, \eta, \xi, t, T ; \theta)$ as

$$
\begin{aligned}
& I(v, x, h, \eta, \xi, t, T ; \theta) \\
& \quad=\log v-\frac{2}{\theta} \log \mathbb{E}^{h, \eta, \xi}\left[\exp \left(\frac{\theta}{2} \int_{0}^{T-t} g(X(s), h(s), \eta(s), \xi(s), r(s+t) ; \theta) \mathrm{d} s\right)\right] .
\end{aligned}
$$

This will lead us to frame the auxiliary game GII that constitutes our first step as stated in Section 1.1.

Step 1. In a worst-case risk-sensitive asset management scenario, the investor chooses a portfolio process $h$ so as to maximize the expected exponential-of-integral performance index $I$. Then the response of the market to this choice is to select $(\eta, \xi)$ (and, hence, a probability measure) that minimizes the maximum expected exponential-of-integral performance index. Formally, the upper value of this game is given by

$$
\bar{u}(t, x)=\sup _{h \in \mathscr{H}(T)} \inf _{(\eta, \xi) \in \mathcal{O}(T)} I(v, x, h, \eta, \xi, t, T ; \theta),
$$

while the lower value of the game is given by

$$
\underline{u}(t, x)=\inf _{(\eta, \xi) \in \mathcal{O}(T)} \sup _{h \in \mathcal{H}(T)} I(v, x, h, \eta, \xi, t, T ; \theta) .
$$

The game has a value provided

$$
\bar{u}(t, x)=\underline{u}(t, x)=u(t, x)=I(v, x, \hat{h}, \hat{\eta}, \hat{\xi}, t, T ; \theta)
$$

and, hence, $\hat{h},(\hat{\eta}, \hat{\xi})$ is a saddle point equilibrium. We aim to provide a verification lemma for which (2.6) is satisfied. In that spirit, consider the exponentially transformed criterion which is simply obtained via the transformation $\tilde{u}(t, x)=\exp (-(\theta / 2) u(t, x))$. This transformation defines what we term game GII. 
Game GII. Obtain $\hat{h} \in \mathcal{H}(T)$ and $(\hat{\eta}, \hat{\xi}) \in \mathcal{O}(T)$ such that

$$
\begin{aligned}
& \tilde{u}(t, x) \\
& =\inf _{h \in \mathcal{H}(T)} \sup _{(\eta, \xi) \in \mathcal{O}(T)} \mathbb{E}^{h, \eta, \xi}\left[\exp \left\{\frac{\theta}{2} \int_{0}^{T-t} g(X(s), h(s), \eta(s), \xi(s), r(s+t) ; \theta) \mathrm{d} s\right\} v^{-\theta / 2}\right] \\
& =\sup _{(\eta, \xi) \in \mathcal{O}(T)} \inf _{h \in \mathcal{H}(T)} \mathbb{E}^{h, \eta, \xi}\left[\exp \left\{\frac{\theta}{2} \int_{0}^{T-t} g(X(s), h(s), \eta(s), \xi(s), r(s+t) ; \theta) \mathrm{d} s\right\} v^{-\theta / 2}\right] \\
& =\mathbb{E}^{\hat{h}, \hat{\eta}, \hat{\xi}}\left[\exp \left\{\frac{\theta}{2} \int_{0}^{T-t} g(X(s), \hat{h}(s), \hat{\eta}(s), \hat{\xi}(s), r(s+t) ; \theta) \mathrm{d} s\right\} v^{-\theta / 2}\right] .
\end{aligned}
$$

\section{An HJBI equation for game GII}

Step 2. Let us now define a couplet process $Y^{h,(\eta, \xi)}(t)$ as

$$
\begin{aligned}
\mathrm{d} Y^{h,(\eta, \xi)}(s) & \\
= & \left(\begin{array}{l}
\mathrm{d} Y_{0}(s) \\
\mathrm{d} Y_{1}(s)
\end{array}\right) \\
= & \left(\begin{array}{c}
\mathrm{d} s \\
\mathrm{~d} X(s)
\end{array}\right) \\
= & \left(\left(b+B X(s)-\Lambda\left(\eta^{\top}(s) X(s)+\xi^{\top}(s)\right)-\frac{\theta}{2} \Lambda \Sigma^{\top} h(s)\right) \mathrm{d} t+\Lambda \mathrm{d} W^{h, \eta, \xi}(s)\right), \\
& Y_{0}(0)=s \in[0, T], \quad Y_{1}(0)=y=\left(y^{1}, \ldots, y^{n}\right) .
\end{aligned}
$$

The control process $h(s)=h(s, \omega)$ is assumed to be Markovian. Then the process $Y^{h,(\eta, \xi)}(s)$ is a Markov process whose generator, acting on a function $\tilde{u}(y) \in C_{0}^{1,2}\left((0, T) \times \mathbb{R}^{n}\right)$, where $C_{0}^{1,2}$ (the space of functions with compact support on $(0, T) \times \mathbb{R}^{n}$ such that it is once continuously differentiable in time and twice continuously differentiable in space with variable $x$ ) is given by

$\tilde{\mathcal{A}}^{h,(\eta, \xi)} \tilde{u}(y)=\frac{\partial \tilde{u}(y)}{\partial s}+\left(b+B x-\Lambda\left(\eta^{\top} x+\xi^{\top}\right)-\frac{\theta}{2} \Lambda \Sigma^{\top} h\right)^{\top} D \tilde{u}(y)+\frac{1}{2} \operatorname{tr}\left(\Lambda \Lambda^{*} D^{2} \tilde{u}(y)\right)$

in which

$$
D \tilde{u}(y) \triangleq\left(\frac{\partial \tilde{u}(y)}{\partial y_{1}^{1}}, \ldots, \frac{\partial \tilde{u}(y)}{\partial y_{1}^{n}}\right)^{\top}
$$

and $D^{2} \tilde{u}(y)$ is the matrix defined as

$$
D^{2} \tilde{u}(y) \triangleq\left[\frac{\partial^{2} \tilde{u}(y)}{\partial y_{1}^{i} \partial y_{1}^{j}}\right], \quad i, j=1,2, \ldots, n .
$$

By an application of the Feynman-Kac formula, it can be deduced that the HJBI PDE for $\tilde{u}(y)$ is given by

$$
\left(\tilde{A}^{\hat{h},(\hat{\eta}, \hat{\xi})}+\frac{\theta}{2} g(x, \hat{h}(y), \hat{\eta}, \hat{\xi}, r ; \theta)\right) \tilde{u}(y)=0 .
$$

In the following proposition, we present a diagnostic to identify a solution to the game GII. 
Proposition 3.1. Define $\&=(0, T) \times \mathbb{R}^{n}$. Let the function $\tilde{w} \in \mathcal{C}^{1,2}(\S) \cap \mathcal{C}(\bar{\S})$. Then there exists a (Markov) control $\hat{h} \in \mathcal{H}(T)$, and $(\hat{\eta}, \hat{\xi}) \in \mathcal{O}(T)$ such that, for each $y \in \mathcal{\&}$,

(i) $\left(\tilde{\mathscr{A}}^{h,(\hat{\eta}, \hat{\xi})}+(\theta / 2) g(x, h, \hat{\eta}, \hat{\xi}, r ; \theta)\right)[(\tilde{w}(y))] \geq 0$ for all $h \in \mathbb{R}^{m}$;

(ii) $\left(\tilde{\mathscr{A}}^{\hat{h}(y),(\eta, \xi)}+(\theta / 2) g(x, \hat{h}(y), \eta, \xi, r ; \theta)\right)[(\tilde{w}(y))] \leq 0$ for all $\eta \in \mathbb{R}^{n \times(n+m)}, \xi \in$ $\mathbb{R}^{1 \times(n+m)} ;$

(iii) $\left(\tilde{\mathscr{A}}^{\hat{h}(y),(\hat{\eta}, \hat{\xi})}+(\theta / 2) g(x, \hat{h}(y), \hat{\eta}, \hat{\xi}, r ; \theta)\right)[(\tilde{w}(y))]=0$;

(iv) $\left[\left(\tilde{w}\left(T, X_{T}\right)\right)\right]=v^{-\theta / 2}$;

(v) $\mathbb{E}^{h, \eta, \xi}\left[\int_{0}^{T-t} D \tilde{w}^{\top}(t+s, X(s)) \Lambda \mathrm{e}^{\tilde{Z}_{s}} \mathrm{~d} W_{s}^{h, \eta, \xi}\right]=0$ for all $h \in \mathbb{R}^{m}, \eta \in \mathbb{R}^{n \times(n+m)}$, and $\xi \in \mathbb{R}^{1 \times(n+m)}$,

where $\tilde{Z}(s)=\tilde{Z}_{s}(h, \eta, \xi):=(\theta / 2)\left\{\int_{0}^{s} g(X(\tau), h(\tau), \eta(\tau), \xi(\tau), r(t+\tau) ; \theta) \mathrm{d} \tau\right\}$. Define

$$
\begin{aligned}
\tilde{I}(v, & x, h, \eta, \xi, t, T ; \theta) \\
& =\exp \left(-\frac{\theta}{2} I(v, x, h, \eta, \xi, t, T ; \theta)\right) \\
& =\mathbb{E}^{h, \eta, \xi}\left[\exp \left\{\frac{\theta}{2} \int_{0}^{T-t} g(X(s), h(s), \eta(s), \xi(s), r(s+t) ; \theta) \mathrm{d} s\right\} v^{-\theta / 2}\right] .
\end{aligned}
$$

Then

$$
\begin{aligned}
\tilde{u}(0, x) & =\tilde{w}(0, x) \\
& =\tilde{I}(v, x, \hat{h}, \hat{\eta}, \hat{\xi}, 0, T ; \theta) \\
& =\inf _{h \in \mathscr{H}(T)}\left\{\sup _{(\eta, \xi) \in \mathcal{O}(T)}[\tilde{I}(v, x, h, \eta, \xi, 0, T ; \theta)]\right\} \\
& =\sup _{(\eta, \xi) \in \mathcal{O}(T)}\left\{\inf _{h \in \mathscr{H}(T)}[\tilde{I}(v, x, h, \eta, \xi, 0, T ; \theta)]\right\} \\
& =\sup _{(\eta, \xi) \in \mathcal{O}(T)} \tilde{I}(v, x, \hat{h},(\eta, \xi), 0, T ; \theta) \\
& =\inf _{h \in \mathscr{H}(T)} \tilde{I}(v, x, h, \hat{\eta}, \hat{\xi}, 0, T ; \theta) \\
& =\tilde{I}(v, x, \hat{h}, \hat{\eta}, \hat{\xi}, 0, T ; \theta)
\end{aligned}
$$

and $(\hat{h},(\hat{\eta}, \hat{\xi}))$ is a saddle point equilibrium.

Proof. Apply Itô's formula to $\tilde{w}(s, X(s)) \mathrm{e}^{\tilde{Z}(s)}$ to obtain

$$
\begin{gathered}
\tilde{w}(T, X(T-t)) \mathrm{e}^{\tilde{Z}(T-t)} \\
=\tilde{w}(t, x)+\int_{0}^{T-t}\left(\left(\tilde{A}^{h, \eta, \xi}+\frac{\theta}{2} g(X(s), h(X(s)), \eta(s), \xi(s), r(s+t) ; \theta)\right)\right. \\
\left.\quad \times \tilde{w}(t+s, X(s))) \mathrm{e}^{\tilde{Z}_{s}}\right) \mathrm{d} s \\
+\int_{0}^{T-t}\left(D \tilde{w}^{\top}(t+s, X(s)) \Lambda\right) \mathrm{e}^{\tilde{Z}(s)} \mathrm{d} W^{h, \eta, \xi}(s) .
\end{gathered}
$$


Taking the expectation with respect to $\mathbb{P}^{h, \eta, \xi}$, from condition (v) of Proposition 3.1 , the stochastic integral in (3.1) vanishes. Now setting $t=0$ and further applying conditions (i) and (iv) of Proposition 3.1, we obtain

$$
\mathbb{E}^{h, \eta, \xi}\left[\tilde{w}\left(T, X_{T}\right) \mathrm{e}^{\tilde{Z}_{T}}\right] \geq \tilde{w}(0, x) .
$$

Since this inequality holds for all $h \in \mathscr{H}(T)$, we have

$$
\inf _{h \in \mathcal{H}(T)} \mathbb{E}^{h, \eta, \xi}\left[v^{-\theta / 2} \mathrm{e}^{\tilde{Z}_{T}}\right] \geq \tilde{w}(0, x) .
$$

Hence, we have

$$
\sup _{(\eta, \xi) \in \mathcal{O}(T)} \inf _{h \in \mathscr{H}(T)} \mathbb{E}^{h, \eta, \xi}\left[v^{-\theta / 2} \mathrm{e}^{\tilde{Z}_{T}}\right] \geq \inf _{h \in \mathcal{H}(T)} \mathbb{E}^{h, \eta, \xi}\left[v^{-\theta / 2} \mathrm{e}^{\tilde{Z}_{T}}\right] \geq \tilde{w}(0, x) .
$$

Similarly, setting $t=0$ and applying conditions (ii), (iv), and (v) of Proposition 3.1, we obtain the following upper value of the game:

$$
\inf _{h \in \mathcal{H}(T)} \sup _{(\eta, \xi) \in \mathcal{O}(T)} \mathbb{E}^{h, \eta, \xi}\left[v^{-\theta / 2} \mathrm{e}^{\tilde{Z}_{T}}\right] \leq \sup _{(\eta, \xi) \in \mathcal{O}(T)} \mathbb{E}^{h, \eta, \xi}\left[v^{-\theta / 2} \mathrm{e}^{\tilde{Z}_{T}}\right] \leq \tilde{w}(0, x) .
$$

Also setting $t=0$ and using conditions (iii), (iv), and (v) of Proposition 3.1, we obtain,

$$
\begin{aligned}
\mathbb{E}^{\hat{h},(\hat{\eta}, \hat{\xi})} & {\left[\tilde{w}\left(T, X_{T}\right) \mathrm{e}^{\tilde{Z}_{T}}\right] } \\
= & \tilde{w}(0, x) \\
= & \mathbb{E}^{\hat{h},(\hat{\eta}, \hat{\xi})}\left[\exp \left\{\frac{\theta}{2} \int_{0}^{T} g(X(s), \hat{h}(X(s)), \hat{\eta}(s), \hat{\xi}(s), r(s) ; \theta) \mathrm{d} s\right\} v^{-\theta / 2}\right] .
\end{aligned}
$$

From (3.2), (3.3), and (3.4), and the fact that

$$
\sup _{(\eta, \xi) \in \mathcal{O}(T)} \inf _{h \in \mathcal{H}(T)}\left[v^{-\theta / 2} \mathrm{e}^{\tilde{Z}_{T}}\right] \leq \inf _{h \in \mathcal{H}(T)} \sup _{(\eta, \xi) \in \mathcal{O}(T)}\left[v^{-\theta / 2} \mathrm{e}^{\tilde{Z}_{T}}\right]
$$

automatically holds, the conclusion now follows.

We now return to the game problem involving $u$ as the payoff function.

Corollary 3.1. It holds that $\underline{u}(0, x)=\bar{u}(0, x)=u(0, x)$.

Proof. The value functions $u$ and $\tilde{u}$ are related through the strictly monotone continuous transformation $\tilde{u}(t, x)=\exp (-(\theta / 2) u(t, x))$. Thus, admissible (optimal) strategies for the exponentially transformed problem $\tilde{u}$ obtained via Proposition 3.1 are also admissible (optimal) for the problem $u$, i.e.

$$
\begin{aligned}
u(0, x) & =\sup _{h \in \mathcal{H}(T)} \inf _{(\eta, \xi) \in \mathcal{O}(T)}\{[I(v, x, h, \eta, \xi, 0, T ; \theta)]\}, \\
& =\inf _{(\eta, \xi) \in \mathcal{O}(T)}\left\{\sup _{h \in \mathcal{H}(T)}[I(v, x, h, \eta, \xi, 0, T ; \theta)]\right\}, \\
& =\inf _{(\eta, \xi) \in \mathcal{O}(T)} I(v, x, \hat{h}, \eta, \xi, 0, T ; \theta), \\
& =\sup _{h \in \mathcal{H}(T)} I(v, x, h, \hat{\eta}, \hat{\xi}, 0, T ; \theta) \\
& =I(v, x, \hat{h}, \hat{\eta}, \hat{\xi}, 0, T ; \theta) .
\end{aligned}
$$

Hence, $\underline{u}(0, x)=\bar{u}(0, x)=u(0, x)$. 


\section{Solving game GII}

Step 3. We seek to find the game payoff function $u$ for the game that will satisfy all the conditions of Proposition 3.1 in terms of $u$. Conditions (i)-(iv) of Proposition 3.1 might be written in the compact form in terms of $u(t, x)$ as

$$
\mathcal{A}^{\hat{h}, \hat{\eta}, \hat{\xi}} u(t, x)=0, \quad u(T, x)=\log v,
$$

where the operator $\mathcal{A}^{h, \eta, \xi} u(t, x)$ for any $h \in \mathbb{R}^{m}$ and $\eta \in \mathbb{R}^{n \times(n+m)}, \xi \in \mathbb{R}^{1 \times(n+m)}$ is given by

$$
\begin{aligned}
\mathcal{A}^{h, \eta, \xi} u(t, x)= & \frac{\partial u(t, x)}{\partial t}+\left(b+B x-\Lambda\left(\eta^{\top}(s) X(s)+\xi^{\top}(s)\right)-\frac{\theta}{2} \Lambda\left(\Sigma^{\top} h\right)\right)^{\top} D u(t, x) \\
& +\frac{1}{2} \operatorname{tr}\left(\Lambda \Lambda^{\top} D^{2} u(t, x)\right) \\
& -\frac{\theta}{4}(D u(t, x))^{\top} \Lambda \Lambda^{\top} D u(t, x)-g(x, h, \eta, \xi, r ; \theta) .
\end{aligned}
$$

The first-order condition for $\hat{h}$ that maximizes $\mathscr{A}^{h, \hat{\eta}, \hat{\xi}}$ over all $\mathscr{H}(T)$ is given by

$$
\hat{h}(t)=\frac{2}{(\theta+2)}\left(\Sigma \Sigma^{\top}\right)^{-1}\left[\delta(t)-\Sigma\left(\hat{\eta}^{\top}(t) X(t)+\hat{\xi}^{\top}\right)-\frac{\theta}{2} \Sigma \Lambda^{\top} D u(t, x)\right] .
$$

Substituting (2.3) into (4.1) yields an expression for the operator $\mathcal{A}^{h, \eta, \xi}$ in $\eta^{\top}(t)$ and $\xi^{\top}(t)$. We minimize $\mathcal{A}^{h, \eta, \xi}$ over the set of controls $\mathcal{O}(T)$. As this operator is linear in continuous and hence bounded controls, $\eta^{\top}(t)$ and $\xi^{\top}(t)$, we guess that the coefficient of the terms $\eta^{\top}(t)$ and $\xi^{\top}(t)$ vanish, leading to

$$
\hat{h}(t)=-\left(\Sigma \Sigma^{\top}\right)^{-1} \Sigma \Lambda^{\top} D u(t, x) .
$$

Motivated by Kuroda and Nagai [10], we will try the functional form for $u$ given by $u(t, x)=$ $\frac{1}{2} x^{\top} Q(t) x+q^{\top}(t) x+k(t)$, where $Q$ is an $n \times n$ symmetric matrix, $q$ is a $n$-element column vector, and $k$ is a scalar. Hence,

$$
\hat{h}(t)=-\left(\Sigma \Sigma^{\top}\right)^{-1} \Sigma \Lambda^{\top}(Q(t) X(t)+q(t)) .
$$

Substituting into (4.2) yields

$$
-\Sigma \Lambda^{\top}(Q(t) X(t)+q(t))=\delta(t)-\Sigma\left(\hat{\eta}^{\top}(t) X(t)+\hat{\xi}^{\top}(t)\right),
$$

which further yields

$\hat{\eta}(t)=\left(Q^{\top}(t) \Lambda \Sigma^{\top}+A^{\top}\right)\left(\Sigma \Sigma^{\top}\right)^{-1} \Sigma, \quad \hat{\xi}(t)=\left((a-r(t) 1)^{\top}+q^{\top}(t) \Lambda \Sigma^{\top}\right)\left(\Sigma \Sigma^{\top}\right)^{-1} \Sigma$.

Thus, $\hat{h}$ is a local maximizing control, and $(\hat{\eta}, \hat{\xi})$ is a local minimizing control that constitutes the saddle point equilibrium for game GII.

Remark 4.1. From Remark 2.1 and (4.4), it can be seen that $\mathbb{P}^{\hat{\eta}}, \hat{\xi}$ is a minimal martingale measure, provided $Q^{\top}(t) \Lambda \Sigma^{\top}\left(\Sigma \Sigma^{\top}\right)^{-1} \Sigma=0$ and $q^{\top}(t) \Lambda \Sigma^{\top}\left(\Sigma \Sigma^{\top}\right)^{-1} \Sigma=0$ for $t \leq T$.

Remark 4.2. From Remark 4.1 and (4.3), it is clear that if the game equilibrium measure corresponds to the minimal martingale measure then the optimal investor strategy satisfies $\hat{h}^{\top}(t) \Sigma=0$. Hence, if the portfolio model does not permit short selling then the optimal investor strategy at game equilibrium is the no-regret strategy, i.e. $(\hat{h}(t)=0)$. 
Remark 4.3. In the case where the factor process and the security (stock) price process has independent noise, i.e. $\Sigma \Lambda^{\top}=0$, then, from Remarks 4.1 and 4.2 , it is obvious that at optimality the worst-case strategy is the no-regret strategy and the factor process always evolves under the minimal martingale measure since the game equilibrium measure is the minimal martingale measure.

As in [10], we can verify that $u(t, x)=\frac{1}{2} x^{\top} Q(t) x+q^{\top}(t) x+k(t)$ satisfies the HJB PDE, i.e. conditions (i)-(iv) of Proposition 3.1 provided

- an $n \times n$ symmetric nonnegative matrix $Q$ satisfies the following matrix-valued Riccati equation:

$$
\frac{\mathrm{d} Q(t)}{\mathrm{d} t}-Q(t) K_{0} Q(t)+K_{1}^{\top} Q(t)+Q(t) K_{1}=0, \quad 0 \leq t \leq T, Q(T)=0,
$$

where

$$
\begin{aligned}
& K_{0}=\frac{\theta}{2} \Lambda\left(I-\frac{\theta-2}{\theta} \Sigma^{\top}\left(\Sigma \Sigma^{\top}\right)^{-1} \Sigma\right) \Lambda^{\top}, \\
& K_{1}=B-\Lambda \eta^{\top}(t)-\Lambda \Sigma^{\top}\left(\Sigma \Sigma^{\top}\right)^{-1} A+\Lambda \Sigma^{\top}\left(\Sigma \Sigma^{\top}\right)^{-1} \Sigma \eta^{\top} .
\end{aligned}
$$

- The $n$-element column vector $q(t)$ satisfies the following linear ODE:

$$
\begin{aligned}
& \frac{\mathrm{d} q(t)}{\mathrm{d} t}+\left(K_{1}^{\top}-Q(t) K_{0}\right) q(t)+Q(t) b-Q^{\top}(t) \Lambda \Sigma^{\top}\left(\Sigma \Sigma^{\top}\right)^{-1}(a-r(t) 1) \\
& +Q^{\top}(t) \Lambda \Sigma^{\top}\left(\Sigma \Sigma^{\top}\right)^{-1} \Sigma \xi^{\top}(t)-Q^{\top}(t) \Lambda \xi^{\top}(t) \\
& \quad=0 \quad \text { for } 0 \leq t \leq T, q(T)=0
\end{aligned}
$$

- and the constant $k(t)$ is a solution to

$$
\begin{aligned}
& \frac{\mathrm{d} k(t)}{\mathrm{d} t}+b^{\top} q(t)+\frac{\theta-2}{4} q^{\top}(t) \Lambda \Sigma^{\top}\left(\Sigma \Sigma^{\top}\right)^{-1} \Sigma \Lambda^{\top} q(t) \\
& +r-q^{\top}(t) \Lambda \Sigma^{\top}\left(\Sigma \Sigma^{\top}\right)^{-1}(a-r(t) 1)+q^{\top}(t) \Lambda \Sigma^{\top}\left(\Sigma \Sigma^{\top}\right)^{-1} \Sigma \xi^{\top}(t) \\
& -\xi(t) \Lambda^{\top} q(t)+\frac{2-\theta}{4} q^{\top}(t) \Lambda \Lambda^{\top} q(t) \\
& \quad=0, \quad \text { for all } 0 \leq t \leq T, k(T)=\log (v) .
\end{aligned}
$$

Condition (iv) of Proposition 3.1 is obvious from the terminal conditions of $Q, q$, and $k$. To show that condition ( $v$ ) of Proposition 3.1 is satisfied by the choice of our payoff function, we need to show that $\mathbb{E}^{h,(\eta, \xi)}\left(<D \tilde{u} \Lambda \mathrm{e}^{Z}, D \tilde{u} \Lambda \mathrm{e}^{Z}>_{t}\right)<\infty$ for all $t \in[0, T]$, where $<\cdot, \cdot>$ as usual symbolizes quadratic co-variation. To show this, we argue as follows. Processes $Q \triangleq(Q(t))_{0 \leq t \leq T}$ and $q \triangleq(q(t))_{0 \leq t \leq T}$ are bounded since they are continuous on the compact support $[0, T]$. By the standard existence-uniqueness argument for a SDE (see Gihman and Skorokhod [7]), $X \in L^{2}\left(\mathbb{P}^{h,(\eta, \xi)}\right)$. Since $D \tilde{u}$ is linear in $X$ with controls $(\eta, \xi)$ assumed bounded, we also have $D \tilde{u} \in L^{2}\left(\mathbb{P}^{h, \eta, \xi}\right)$. To complete the argument it remains to show that $\tilde{u}$ is bounded, which we show now.

Lemma 4.1. It holds that $0<\tilde{u}<\exp \left(-(\theta / 2) \int_{0}^{T-t} r(s+t) \mathrm{d} s\right) v^{-\theta / 2}$.

Proof. From the definition of $\tilde{u}$ in (2.7), for any optimal control $\mathcal{O}(T)$, the strategy $\hat{h}(t)=0$ for $t \leq T$ is suboptimal, and, hence, will provide an upper bound on $\tilde{u}$. Furthermore, for 
the special case when $\eta(t)=\xi(t)=0$ for any $h \in \mathcal{H}(T)$ and $t \leq T$ corresponds to the 'no-model-uncertainty' case for which we now obtain an upper bound on $\tilde{u}$. Formally, we write these statements as

$$
\begin{aligned}
\tilde{u}(t, x) & \left.=\inf _{h \in \mathcal{H}(T)} \mathbb{E}^{h, \hat{\eta}, \hat{\xi}}\left[\exp \left\{\frac{\theta}{2} \int_{0}^{T-t} g(X(s), h(s), \hat{\eta}(s), \hat{\xi} s), r(s+t) ; \theta\right) \mathrm{d} s\right\} v^{-\theta / 2}\right] \\
& \leq \mathbb{E}^{0, \hat{\eta}, \hat{\xi}}\left[\exp \left\{\frac{\theta}{2} \int_{0}^{T-t} g(X(s), 0, \hat{\eta}(s), \hat{\xi}(s), r(s+t) ; \theta) \mathrm{d} s\right\} v^{-\theta / 2}\right] \\
& \leq \mathbb{E}^{0,0,0}\left[\exp \left\{\frac{\theta}{2} \int_{0}^{T-t} g(X(s), 0,0,0, r(s+t) ; \theta) \mathrm{d} s\right\} v^{-\theta / 2}\right] \\
& =\exp \left(-\frac{\theta}{2} \int_{0}^{T-t} r(s+t) \mathrm{d} s\right) v^{-\theta / 2} .
\end{aligned}
$$

Hence, the conclusion follows.

We now formalize the solution to this game GI.

Step 4. We first show that the controls belonging to $\mathcal{H}(T)$ and $\mathcal{O}(T)$ satisfy the following change of measure criterion.

Lemma 4.2. From the choice of the probability space of the controls $h \in \mathcal{H}(T)$ and $(\eta, \xi) \in$ $\mathcal{O}(T)$, we have

$$
\mathbb{E}\left[\mathcal{E}\left(-\frac{\theta}{2} \int_{0}\left[(Q(t) X(t)+q(t)) \Lambda+h^{\top}(t) \Sigma\right] \mathrm{d} W^{\eta, \xi}(t)\right)_{T}\right]=1 .
$$

Proof. The above result holds if the following Kazamaki condition is satisfied:

$$
\mathbb{E}\left[\exp \left(\int_{0}^{t} \theta\left(\frac{(Q(s) X(s)+q(s)) \Lambda+h^{\top}(s) \Sigma}{2}\right) \mathrm{d} W^{\eta, \xi}(s)\right)\right]<\infty \quad \text { for all } t \in[0, T] .
$$

By an application of the Cauchy-Schwartz inequality, we have

$$
\begin{aligned}
\mathbb{E}[\exp & \left.\left(\int_{0}^{t} \theta\left(\frac{(Q(s) X(s)+q(s)) \Lambda+\left(h^{\top}(s) \Sigma\right)}{2}\right) \mathrm{d} W^{\eta, \xi}(s)\right)\right] \\
\leq & \left(\mathbb{E}\left[\exp \left(\int_{0}^{t} \theta(Q(s) X(s)+q(s)) \Lambda \mathrm{d} W^{\eta, \xi}(s)\right)\right]\right)^{1 / 2} \\
& \times\left(\mathbb{E}\left[\exp \left(\int_{0}^{t} \theta\left(h^{\top}(s) \Sigma\right) \mathrm{d} W^{\eta, \xi}(s)\right)\right]\right)^{1 / 2} \text { for } t \in[0, T] .
\end{aligned}
$$

Since $X$ is a Gaussian process, mimicking arguments similar to Lemma 2.1, it follows that

$$
\left(\mathbb{E}\left[\exp \left(\int_{0}^{t} \theta(Q(s) X(s)+q(s)) \Lambda \mathrm{d} W_{s}^{\eta, \xi}\right)\right]\right)^{1 / 2}<\infty \text { for all } t \in[0, T] .
$$

From the assumption on the probability space of the controls $\mathscr{H}(T)$, we conclude that

$$
\left(\mathbb{E}\left[\exp \left(\int_{0}^{t} \theta\left(h^{\top}(s) \Sigma\right) \mathrm{d} W^{\eta, \xi}(s)\right)\right]\right)^{1 / 2}<\infty \quad \text { for } t \in[0, T] .
$$

Hence, the Kazamaki condition holds and the conclusion follows. 
We now show that the saddle point equilibrium controls obtained by solving game GII is in fact also a saddle point equilibrium for the original game problem GI.

Proposition 4.1. If there exists a solution $Q$ to the matrix-valued Riccati equation (4.5), then the saddle point equilibrium strategies $\hat{h}$ and $(\hat{\eta}, \hat{\xi})$ obtained from (4.3) and (4.4), respectively, as a result of solving the auxiliary game GII where $q$ is a solution of (4.6) and $k$ is a solution of (4.7) is in fact also the saddle point equilibrium for the finite-horizon game GI, namely

$$
\begin{aligned}
\sup _{h \in \hat{\mathscr{H}}(T)} \inf _{(\eta, \xi) \in \hat{\mathcal{O}}(T)} \tilde{J}(v, h, \eta, \xi, T ; \theta) & =\inf _{(\eta, \xi) \in \hat{\mathcal{O}}(T)} \sup _{h \in \hat{\mathscr{H}}(T)} \tilde{J}(v, h, \eta, \xi, T ; \theta), \\
& =\tilde{J}(v, \hat{h}, \hat{\eta}, \hat{\xi}, T ; \theta,) \\
& =\frac{1}{2} x^{\top} Q(0) x+q^{\top}(0) x+k(0),
\end{aligned}
$$

where

Proof. Define

$$
\tilde{J}(v, h, \eta, \xi, T ; \theta) \triangleq \frac{-2}{\theta} \log \mathbb{E}^{\eta, \xi}\left[\exp \left[\frac{-\theta}{2} \log V^{h, \eta, \xi}(T)\right]\right] .
$$

$$
\begin{aligned}
\bar{Z}_{s}= & \bar{Z}_{s}(h, \eta, \xi) \\
= & \frac{\theta}{2}\left\{\int_{0}^{s} g(X(\tau), h(\tau), \eta(\tau), \xi(\tau), r(t+\tau) ; \theta) \mathrm{d} \tau-\left(h^{\top}(\tau) \Sigma\right) \mathrm{d} W^{\eta, \xi}(\tau)\right. \\
& \left.\quad-\frac{\theta}{4}\left(h^{\top}(\tau) \Sigma\right)^{\top}\left(h^{\top}(\tau) \Sigma\right) \mathrm{d} \tau\right\} .
\end{aligned}
$$

Also define $\chi(t, x)=-(\theta / 2)(u(t, x)-\log v)$. From some straightforward calculations provided in Appendix A, we obtain the following relation:

$$
\begin{aligned}
& \exp \{\chi(T, X(T-t))+\bar{Z}(T-t)\} \\
&=\exp (\chi(t, x)) \exp \left[\int_{0}^{T-t}-\frac{\theta}{2}\left(\mathcal{A}^{h, \eta, \xi} u\left(t+s, X_{s}\right)\right) \mathrm{d} s\right. \\
&-\int_{0}^{T-t} \frac{\theta}{2}\left[D u\left(t+s, X_{s}\right)^{\top} \Lambda+\left(h^{\top}(t) \Sigma\right)\right] \mathrm{d} W_{t}^{\eta, \xi} \\
&-\int_{0}^{T-t} \frac{\theta^{2}}{8}\left[D u\left(t+s, X_{S}\right)^{\top}+\left(h^{\top}(t) \Sigma\right)\right] \\
&\left.\times\left[D u\left(t+s, X_{S}\right)^{\top}+h^{\top}(t) \Sigma\right]^{\top} \mathrm{d} s\right] .
\end{aligned}
$$

We have shown that the saddle point equilibrium strategies $\hat{h}$ and $(\hat{\eta}, \hat{\xi})$ deduced by solving game GI with corresponding game payoff function $u$ satisfies conditions (i)-(v) of Proposition 3.1. Therefore, from condition (iv), we have $\chi(T, x)=0$. Moreover, $\left(V^{h, \eta, \xi}(T)\right)^{-\theta / 2}=$ $v^{-\theta / 2} \mathrm{e}^{\bar{Z}_{T}}$. Setting $t=0$ and taking condition (i) into account for $\eta=\hat{\eta}, \xi=\hat{\xi}$, and for any $h \in \hat{\mathscr{H}}(T)$, from (4.8), we have

$$
\begin{aligned}
\left(V^{h, \eta, \xi}(T)\right)^{-\theta / 2} & \\
\geq \mathrm{e}^{-(\theta / 2) u(0, x)} \exp [ & -\int_{0}^{T} \frac{\theta}{2}\left[D u(s, X(s))^{\top} \Lambda+h^{\top}(s) \Sigma\right] \mathrm{d} W^{\eta, \xi}(s) \\
& \left.-\int_{0}^{T} \frac{\theta^{2}}{8}\left[D u(s, X(s))^{\top}+h^{\top}(s) \Sigma\right]\left[D u(s, X(s))^{\top}+h^{\top}(s) \Sigma\right]^{\top} \mathrm{d} s\right] .
\end{aligned}
$$


Now, by taking expectations with respect to the physical probability measure $\mathbb{P}^{\eta, \xi}$ on both sides of above equation and using Lemma 4.2, we obtain

$$
\tilde{J}(v, h, \eta, \xi, T ; \theta) \leq u(0, x) .
$$

This inequality holds for all $h \in \mathscr{H}(T)$. Hence, we have

$$
\sup _{h \in \mathscr{H}(T)} \tilde{J}(v, h, \eta, \xi, T ; \theta) \leq u(0, x) .
$$

Hence, we have

$$
\inf _{(\eta, \xi) \in \mathcal{O}(T)} \sup _{h \in \mathscr{H}(T)} \tilde{J}(v, h, \eta, \xi, T ; \theta) \leq \sup _{h \in \mathscr{H}(T)} \tilde{J}(v, h, \eta, \xi, T ; \theta) \leq u(0, x) .
$$

Likewise, setting $t=0$ and taking conditions (ii) and (iv) into account, we have

$$
\sup _{h \in \mathscr{H}(T)} \inf _{(\eta, \xi) \in \mathcal{O}(T)} \tilde{J}(v, h, \eta, \xi, T ; \theta) \geq u(0, x) \geq \inf _{(\eta, \xi) \in \mathcal{O}(T)} \sup _{h \in \mathscr{H}(T)} \tilde{J}(v, h, \eta, \xi, T ; \theta) .
$$

Similarly, setting $t=0$ and taking conditions (iii) and (v) into account for $h=\hat{h}, \gamma=\hat{\gamma}$ such that $\hat{h} \in \mathscr{H}(T)$ and $(\hat{\eta}, \hat{\xi}) \in \mathcal{O}(T)$, we obtain

$$
\tilde{J}(v, \hat{h}, \hat{\eta}, \hat{\xi}, T ; \theta)=u(0, x) .
$$

From (4.10)-(4.12) and the fact that

$$
\sup _{h \in \mathscr{H}(T)} \inf _{(\eta, \xi) \in \mathcal{O}(T)} \tilde{J}(v, h, \eta, \xi, T ; \theta) \leq \inf _{(\eta, \xi) \in \mathcal{O}(T)} \sup _{h \in \mathscr{H}(T)} \tilde{J}(v, h, \eta, \xi, T ; \theta)
$$

automatically holds, we conclude that the saddle point equilibrium controls obtained by solving game GII in fact also constitutes a saddle point strategy for the original game GI.

\section{Appendix A.}

Part proof of Proposition 4.1. Let $\chi(t, x)=-(\theta / 2)(u(t, x)-\log v)$ and

$$
L u(t, x)=\frac{1}{2} \operatorname{tr}\left(\Lambda \Lambda^{\top} D^{2} u(t, x)\right)+\left(b+B x-\Lambda\left(\eta^{\top} x+\xi^{\top}\right)\right)^{\top} D u(t, x) .
$$

Hence, we have

$$
\mathrm{d} \chi(t+s, X(s))=-\frac{\theta}{2}\left(\frac{\partial u}{\partial t}+L u\right)(t+s, X(s)) \mathrm{d} s-\frac{\theta}{2} D u(t+s, X(s))^{\top} \Lambda \mathrm{d} W^{\eta, \xi}(s) .
$$

Therefore,

$$
\begin{aligned}
\frac{\mathrm{d} \exp \{\chi(t+s, X(s))\}}{\exp \{\chi(t+s, X(s))\}} & \\
= & -\frac{\theta}{2}\left(\frac{\partial u}{\partial t}(t, x)+L u\right)(t+s, X(s))-\frac{\theta}{2} D u(t+s, X(s))^{\top} \Lambda \mathrm{d} W^{\eta, \xi}(s) \\
& +\frac{\theta^{2}}{8} D u^{\top} \Lambda \Lambda^{\top} D u(t+s, X(s)) \mathrm{d} s .
\end{aligned}
$$


It follows that

$$
\begin{aligned}
\frac{\mathrm{d} \exp \{\chi(t+s, X(s))\} \exp \{Z(s)\}}{\exp \{\chi(t+s, X(s))\} \exp \{Z(s)\}} \\
=-\frac{\theta}{2}\left(\frac{\partial u}{\partial t}(t, x)+L u\right)(t+s, X(s))-\frac{\theta}{2} D u(t+s, X(s))^{\top} \Lambda \mathrm{d} W^{\eta, \xi}(s) \\
\quad+\frac{\theta^{2}}{8} D u^{\top} \Lambda \Lambda^{\top} D u(t+s, X(s)) \mathrm{d} s+\frac{\theta}{2} g(X(t), h(t), \eta(t), \xi(t), r(s+t) ; \theta) \mathrm{d} s \\
\quad-\frac{\theta}{2} h^{\top}(s) \Sigma \mathrm{d} W^{\eta, \xi}(s)+\frac{\theta^{2}}{4} h^{\top}(s) \Sigma \Lambda^{\top} D u(t+s, X(s)) \mathrm{d} s .
\end{aligned}
$$

Integrating the above equation yields (4.9).

\section{Acknowledgement}

The financial support of a Chancellor's Scholarship of the University of Warwick is gratefully acknowledged. This paper is dedicated as an ode to the rich legacy of research work of Wendell H. Fleming [2] and Peter Whittle [12].

\section{References}

[1] Bensoussan, A. (1992). Stochastic Control of Partially Observable Systems. Cambridge University Press.

[2] Berkovitz, L. D., Shreve, S. E. And Ziemer, W. P. (1993). A tribute to Wendell H. Fleming. J. SIAM Control Optimization 31, 273-281.

[3] Bielecki, T. R. And Pliska, S. R. (1999). Risk-sensitive dynamic asset management. Appl. Math. Optimization 39, 337-360.

[4] Fleming, W. H. (1995). Optimal investment models and risk sensitive stochastic control. In Mathematical Finance (IMA Vol. Math. Appl. 65), Springer, New York, pp. 75-88.

[5] Fleming, W. H. and Sheu, S. J. (2002). Risk-sensitive control and an optimal investment model. II. Ann. Appl. Prob. 12, 730-767.

[6] Föllmer, H. AND SchweIzer, M. (2010). Minimal martingale measure. In Encyclopedia of Quantitative Finance, John Wiley, New York, pp. 1200-1204.

[7] Gīhman, İ. İ. And Skorohod, A. V. (1972). Stochastic Differential Equations. Springer, New York.

[8] Girsanov, I. V. (1960). On transforming a certain class of stochastic processes by absolutely continuous substitution of measures. Theory Prob. Appl. 5, 285-301.

[9] Karatzas, I. And Shreve, S. E. (1998). Methods of Mathematical Finance. Springer, New York.

[10] Kuroda, K. And Nagai, H. (2002). Risk-sensitive portfolio optimization on infinite time horizon. Stoch. Stoch. Reports 73, 309-331.

[11] Lefebvre, M. and Montulet, P. (1994). Risk-sensitive optimal investment policy. Internat. J. Systems Sci. 25, 183-192.

[12] Whittle, P. (1990). Risk-Sensitive Optimal Control. John Wiley, Chichester. 